\title{
Motion Estimation Applied to Reconstruct Undersampled Dynamic MRI
}

\author{
Claudia Prieto ${ }^{1}$, Marcelo Guarini ${ }^{1}$, Joseph Hajnal $^{2}$, and Pablo Irarrazaval ${ }^{1}$ \\ ${ }^{1}$ Pontificia Universidad Católica de Chile, \\ Departamento de Ingenieria Eléctrica, Vicuna Mackenna 4860, Chile \\ pim@ing.puc.cl \\ ${ }^{2}$ Hammersmith Hospital, Imperial College London, \\ Du Cane Road W12 ONN, UK
}

\begin{abstract}
Magnetic Resonance Imaging (MRI) has become an important tool for dynamic clinical studies. Regrettably, the long acquisition time is still a challenge in dynamic MRI. Several undersampled reconstruction techniques have been developed to speed up the acquisition without significantly compromising image quality. Most of these methods are based on modeling the pixel intensity changes. Recently, we introduced a new approach based on the motion estimation of each object element (obel, a piece of tissue). Although the method works well, the outcome is a trade off between the maximum undersampling factor and the motion estimation accuracy. In this work we propose to improve its performance through the use of additional data from multiple coils acquisition. Preliminary results on cardiac MRI show that further undersampling and/or improved reconstruction accuracy is achieved using this technique. Furthermore, an approximation of the vector field of motion is obtained. This method is appropriate for sequences where the obels' intensity through time is nearly constant.
\end{abstract}

Keywords: motion estimation, MRI, dynamic images, undersampling.

\section{Introduction}

Over the last years, Magnetic Resonance Imaging (MRI) has become an important tool for dynamic clinical studies. Applications are wide ranging, including cardiac MRI [1]-2], real-time interventional imaging [3] and kinematics of joints 4. The long time involved in the acquisition of an image sequence is still a challenge in dynamic MRI. An active line of research in this area has been aimed to speed up the information capture phase, without significantly compromising the image quality.

The MRI signal corresponds to the Fourier transform of the imaged object density map, along a given sample path. The frequency space where data is acquired is called $k$-space. The way in which this space is covered during the acquisition phase is known as $k$-space trajectory. The ideal approach to acquired dynamic objects would be to collect the fully sampled $k$-space repeatedly for

D. Mery and L. Rueda (Eds.): PSIVT 2007, LNCS 4872, pp. 522 532, 2007.

(C) Springer-Verlag Berlin Heidelberg 2007 
each time frame ( $k$ - $t$ space [5]) and then to reconstruct the desired number of image frames independently. However, in practice the temporal resolution depends on the speed of acquisition of the $k$-space for each frame, which is limited by hardware constraint: 1 . There is a little scope for improving temporal resolution through hardware advancement. As a consequence, considerable research is being aimed towards undersampling reconstruction techniques in $k$-space or $k$ - $t$ space. These techniques reduce the sampling by a certain factor, and later the aliasing artifacts are reduced by estimating the missing data from prior information or through the use of information redundancy in dynamic MRI.

Traditional methods to reconstruct undersampled dynamic images use models based on time varying pixel intensities or are based on temporal frequencies to recover the non acquired data. Among others, those methods include keyhole [6][7, reduced field of view [8], unaliasing by Fourier-encoding the overlaps using the temporal dimension (UNFOLD) [9], $k$ - $t$ broad-use linear acquisition speedup technique ( $k$ - $t$ BLAST) [10, reconstruction employing temporal registration 11. Recently we have introduced a method which recovers the non acquired data by estimating the motion of each object element or obel [12. An obel is defined as a piece of tissue of the object whose intensity remains constant over time. The supporting assumption for this method is that the displacement of an obel has lower bandwidth than the intensity changes through time of a stationary pixel, and therefore it can be described with fewer parameters. A pictorial description of this idea, for a pixel near the edge of a dynamic object, is depicted in Fig 1 .

The proposed method performs quite well, although there is a trade off between the undersampling factor and the accuracy of the motion estimation. A known technique to speed up acquisition in MRI is to use multiple receiver coils in parallel scan time (parallel imaging) as a complementary encoding [13] - [14]. In this work we propose to use the additional information provided by multiple coils to increase the available data, in a scheme that combines the above method based on obels with parallel imaging. The additional information can be used to reach higher undersampling factors or to improve the motion estimation.

Here we describe the proposed method and the preliminary results of applying it to cardiac images. We begin summarizing the basics of the reconstruction technique based on the motion estimation of obels for single coil images. Then, the concept is extended to incorporate parallel imaging by exploiting the additional data from multiple receiver coils. Finally, we provide an analysis of results showing the method potential.

\section{Reconstruction by Obels in Single Coil Acquisition}

Considering that an obel does not change its intensity over time, it is possible to reconstruct any frame of a dynamic sequence from a reference frame and the motion model of each obel initially defined in this frame. This statement is also valid for undersampled dynamic images. Solving the inverse problem from the

\footnotetext{
${ }^{1}$ The gradient strength and slew rate of modern MR scanners is limited by safety concerns related to peripheral nerve stimulation and by the cost associated.
} 


\section{Pixel Intensity Change}

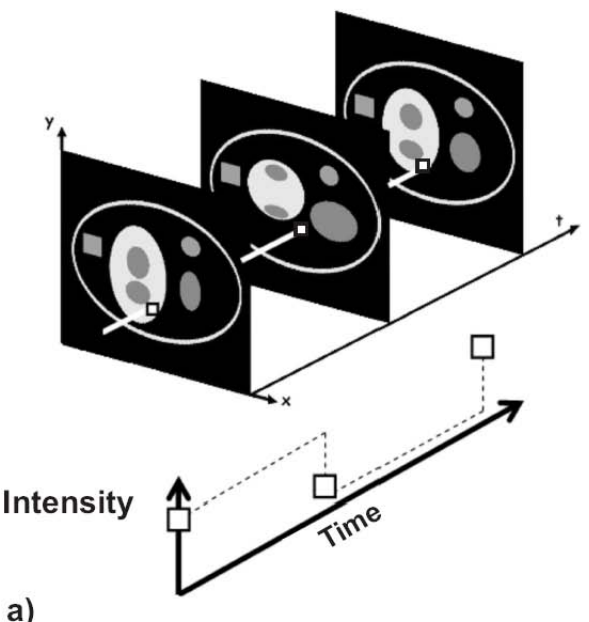

\section{Obel Displacement}

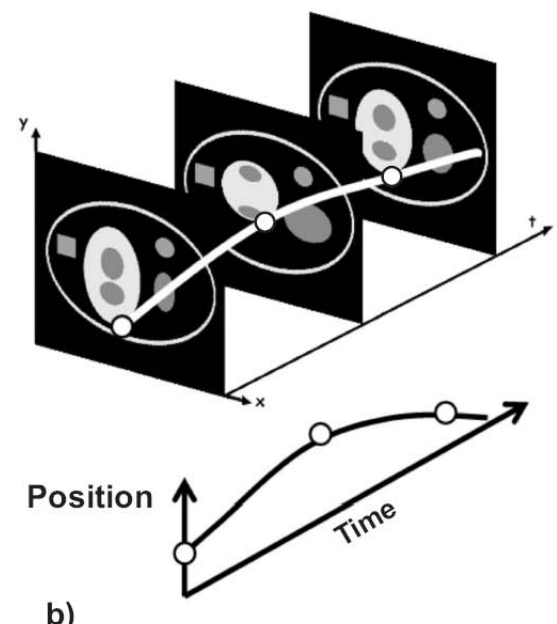

Fig. 1. Comparison between the intensity fluctuation of a pixel in the edge of an object and the displacement of the obel at the same starting position. a) Pixel intensity fluctuation through time. b) Obel's displacement.

undersampled $k$ - $t$ space, a fully sampled reference frame and the motion model of the sequence are obtained, allowing the reconstruction of a fully sampled dynamic sequence.

For simplicity, we first review the fully sampled case. Let $\mathbf{m}_{0}$ be the reference frame and $\mathbf{P}_{t}, t=1 \ldots N_{t}$ the matrix that describes the spatial displacement over time for each obel initially defined in $\mathbf{m}_{0}$. In this way, any frame $\mathbf{m}_{t}$ of the dynamic sequence can be computed using

$$
\mathbf{m}_{t}=\mathbf{P}_{t} \mathbf{m}_{0}
$$

where $\mathbf{P}_{t}$ represents both, the permutation and the interpolation matrices [15]. Since this matrix is large and seldom invertible, a more efficient implementation of the image transformation is achieved by representing it as a spatial transformation

$$
\mathbf{F}_{t}(\mathbf{x})=\mathbf{x}+\mathbf{u}_{t}(\mathbf{x})
$$

where $\mathbf{x}$ is the position vector defined in the coordinate system of $\mathbf{m}_{0}$, and $\mathbf{u}_{t}$ holds the obels' displacement in the image dimensions for each time frame $t$.

Employing this spatial transformation Eq1 becomes

$$
m_{t}(\mathbf{y})=\mathbf{P}_{t} m_{0}(\mathbf{x})=m_{0}\left(\mathbf{F}_{t}^{-1}(\mathbf{y})\right)
$$

where $\mathbf{y}$ is the position vector in any frame of the dynamic sequence $\mathbf{m}_{t}$, in contrast to $\mathbf{x}$ which is defined in $\mathbf{m}_{\mathbf{0}}$. 
Since we need to model the displacement for each obel using few parameteres, we do not use $\mathbf{F}_{t}(\mathbf{x})$ for each time frame $t$, but the parameterised version, $\mathbf{F}(\mathbf{e})$. Each row of the matrix e corresponds to a vector of parameters that describes the displacement of one particular obel initially defined in $\mathbf{m}_{0}$. Now, we can write $m_{t}\left(\mathbf{m}_{0}, \mathbf{e}\right)$ to state that it is possible to reconstruct any frame of the dynamic sequence from a reference frame $\mathbf{m}_{0}$ and a set of parameters $\mathbf{e}$.

This representation can be applied to undersampled dynamic images. As the data in MRI is acquired in the $k$-space, we multiply the dynamic sequence $\mathbf{m}_{t}$ by the Fourier transform $\mathbf{W}$ obtaining

$$
\mathbf{W} m_{t}(\mathbf{y})=\mathbf{W} m_{t}\left(\mathbf{m}_{0}, \mathbf{e}\right)
$$

The desired samples are collected from the $k$-space using an undersampling pattern $S_{t}(\mathbf{k})$. This matrix has elements one and zero indicating if a sample in the position $\mathbf{k}$ of the $k$-space was or was not collected at time frame $t$, respectively. Let $B_{t}(\mathbf{k})$ represent the samples acquired in $k$ - $t$ space, thus

$$
B_{t}(\mathbf{k})=S_{t}(\mathbf{k}) \mathbf{W} m_{t}\left(\mathbf{m}_{0}, \mathbf{e}\right)
$$

which represents a non-linear system. The equations correspond to the acquired samples $B_{t}(\mathbf{k})$, and the unknowns correspond to the reference frame $\mathbf{m}_{\mathbf{0}}$ and the parameteres needed to model the obels' motion e.

In order to improve solution stability the system in Eq 5 is solved in the image domain rather than in the $k$-space domain. To bring the data to the image domain we multiply $B_{t}(\mathbf{k})$ by the inverse Fourier transforms $\mathbf{W}^{H}$, thus

$$
b_{t}(\mathbf{y})=\mathbf{W}^{H} S_{t}(\mathbf{k}) \mathbf{W} m_{t}\left(\mathbf{m}_{0}, \mathbf{e}\right)
$$

where $b_{t}(\mathbf{y})$ is the acquired aliased data.

Let $\mathbf{N}_{d}$ be the spatial dimensions of the image sequence, $\mathbf{N}_{d}=\left(N_{x}, N_{y}\right)$ if the sequence is bidimensional (2D) and $\mathbf{N}_{d}=\left(N_{x}, N_{y}, N_{z}\right)$ if it is three-dimensional (3D). Letting $N_{t}$ be the temporal dimension of the image sequence, $N_{e}$ the size of the matrix e and $Q$ the undersampling factor, conceptually the system becomes fully determined if the following relation holds

$$
\frac{N_{d} N_{t}}{Q} \geq N_{d}+N_{e}
$$

It defines an upper bound for the undersampling factor, given the image sequence size and the degrees of freedom required to model the obels' motion over all the image dimensions. Therefore, there is a trade off between the undersamplig factor $Q$ and the accuracy of the movement estimation given by $N_{e}$. This trade off becomes less critical using multiple coils, as we describe in the next section.

\section{Reconstruction by Obels in Multiple-Coil Acquisition}

In single coil acquisitions of MRI, the imaged object properties are codified using a magnetic field gradient, allowing to collect only one $k$-space sample at a time. 
Complementary encoding can be achieved by employing multiple receiver coils. It is possible because the signal read by each coil varies appreciably with its relative position over the object. In this way, the information about the spatial sensitivity of each receiver can be use to obtain more than one sample at a time [13].

The spatial sensitivity of each receiver does not depend on the imaged object and thus the unknows of our method (described in the previous section) do not change with coil sensitivities. Therefore, it is possible to use the extra information from multiple coils in our method based on obels without modifying the amount of unknowns of the reconstruction process.

Let $\mathbf{C}_{i}$ be the sensitivity of coil $i, i=1 \ldots N_{c}$, where $N_{c}$ is the number of parallel coils considered. Let $\mathbf{m}_{t, i}$ the image acquired with coil $i$ at the time frame $t$, thus

$$
\mathbf{m}_{t, i}=\mathbf{C}_{i} \mathbf{m}_{t}
$$

Repeating the procedure described in the previous section for each single coil image, $\mathbf{m}_{t, i}$, we obtain,

$$
b_{t, i}(\mathbf{y})=\mathbf{W}^{H} S_{t}(\mathbf{k}) \mathbf{W} \mathbf{C}_{i} m_{t}\left(\mathbf{m}_{0}, \mathbf{e}\right)
$$

where $\mathbf{b}_{t, i}$ represents the aliased single coil data. We can obtain the image sequence $\mathbf{m}_{t}$ from the data acquired with each receiver $\mathbf{b}_{t, i}$ solving the non-linear system Eq.9. The advantage of this approach is that the system has more equations and the same number of unknowns as in the single coil case. The system becomes fully determined if

$$
\frac{N_{d} N_{t} N_{c}}{Q} \geq N_{d}+N_{e}
$$

Clearly then, the higher the number of coils, $N_{c}$, the higher the upper bound for the undersampling factor $Q$ or for the parameters of the motion model $N_{e}$.

We can solve Eq9 setting it as an optimization problem. Let the sum over all the coils and time frames of the difference between the acquired and the estimated data be the cost function to be minimized, then

$$
\min \Delta b=\sum_{t=1}^{N_{t}} \sum_{i=1}^{N_{c}}\left\|b_{t, i}(\mathbf{y})-\hat{b}_{t, i}(\mathbf{y})\right\|_{2}
$$

where

$$
\hat{b}_{t, i}(\mathbf{y})=\mathbf{W}^{H} S_{t}(\mathbf{k}) \mathbf{W} \mathbf{C}_{i} m_{t}\left(\hat{\mathbf{m}}_{0}, \hat{\mathbf{e}}\right)
$$

The problem can be solved by two nested optimization loops. In the inner loop, $\mathbf{e}$ is considered known and $\Delta b$ is minimized as a function of $\mathbf{m}_{0}$. In the outer loop the inner estimation of $\mathbf{m}_{0}$ is known and the minimum of $\Delta b$ is found as a function of $\mathbf{e}$. After convergence, $\mathbf{m}_{\mathbf{0}}$ and $\mathbf{e}$ are found, representing the best fitted model for the acquired multiple coil data. 


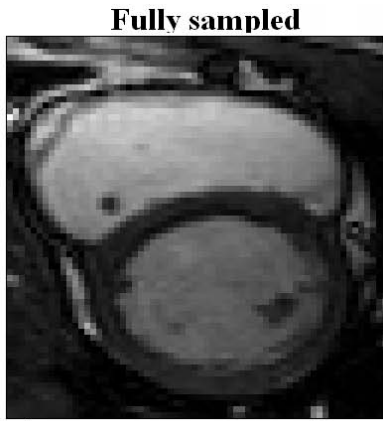

a)

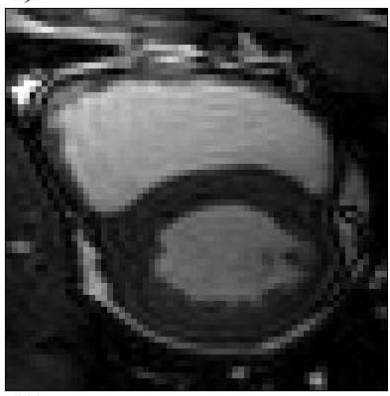

d) 8x Our method, 5 coils

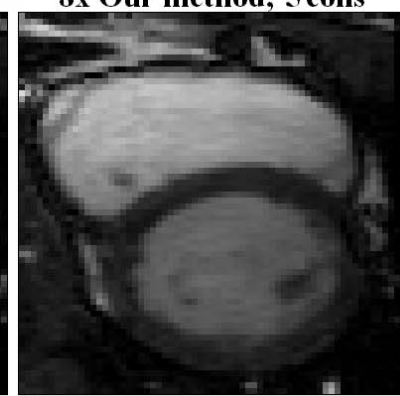

b)

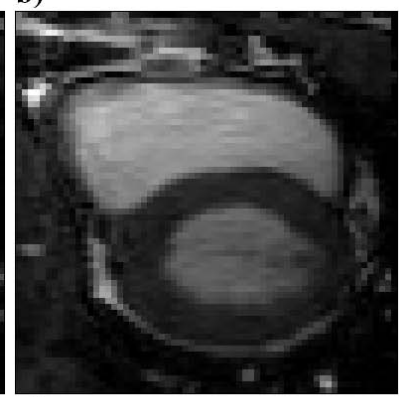

e) 8x Our method, 1coil

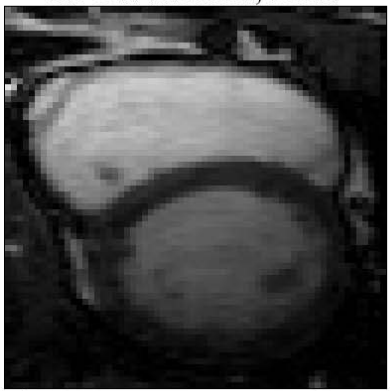

c)

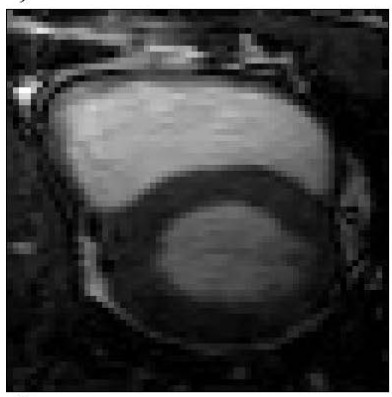

f)

Fig. 2. Reconstructed images from undersampled data using motion estimation. 2D short-axis cardiac sequence with a post-acquisition undersampling factor of 8 . Frames 4 and 27 of a 50 -frame sequence are shown. a) Fully sampled frame 4. b) Reconstructed image frame 4 with our method from a 5-coil acquisition. c) Reconstructed image with our method frame 4 from a 1-coil acquisition. d) Fully sampled frame 27. b) Reconstructed image frame 27 with our method from a 5-coil acquisition. c) Reconstructed image frame 27 with our method from a 1-coil acquisition.

\section{Experimental Results}

The proposed algorithm was used to reconstruct a $2 \mathrm{D}$ cardiac sequence. A fully sampled sequence was collected on a Philips Intera $1.5 \mathrm{~T}$ with a 5-channel cardiac coil. The acquired raw data was undersampled post-acquisition by factors of 8 and 16. The images were reconstructed considering two reference frames, $\mathbf{m}_{0}$ and $\mathbf{m}_{c}$, at the beginning and at the middle of the cardiac sequence. Each pixel in $\mathbf{m}_{0}$ was considered an obel, and B-Splines with three coefficients were used to describe their displacement in every Cartesian direction through time. For an undersampling of 8 , the results of the single-coil method were compared against those obtained using the multiple-coil approach. The results of the proposed method for an undersampling factor of 16 were only compared with those obtained using sliding window SW reconstruction [16]. This level of undersampling is not possible using the method based on obels with a single coil because of constraints in the amount of data (Eq,7). 


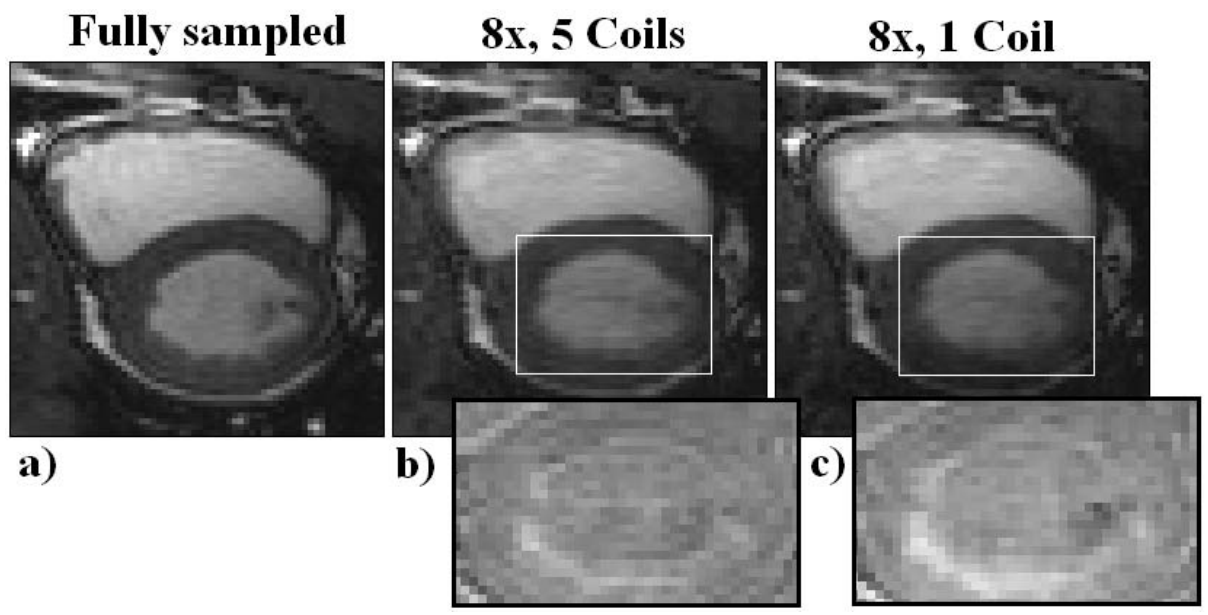

Fig. 3. Difference images. 2D short-axis cardiac sequence with a post-acquisition undersampling factor of 8 . The images show the differences between the fully sampled and the reconstructed images. a) Fully sampled image. b) Our reconstruction using multiple coils and the difference respect to the fully sampled image. c) Our reconstruction using single-coil and the difference respect to the fully sampled image.

\subsection{Method}

A steady-state free precession (SSFP) cardiac sequence was aquired with the following scanning parameters: 2D balanced fast field echo (B-FFE) cardiac gated, short-axis acquisition, $\mathrm{TR} / \mathrm{TE}=3 \mathrm{~ms} / 1.46 \mathrm{~ms}$, flip angle $=50^{\circ}, \mathrm{FOV}$ $=400 \times 320 \mathrm{~mm}^{2}$, resolution $=1.56 \times 2.08 \mathrm{~mm}^{2}$, slice thickness $=8 \mathrm{~mm}$, acquisition matrix $=256 \times 154,50$ frames, five channel sinergy coil and breath-hold duration close to 25 seconds.

The acquired data was undersampled post-acquisition by factors of 8 and 16 using a lattice pattern (similarly to the one used in [10]). This pattern allows to sample $1 / Q$ of the samples available in the $k$-space.

Two references frames $\left(\mathbf{m}_{0}\right.$ and $\left.\mathbf{m}_{c}\right)$ were considered to reconstruct the dynamic sequence, thus the optimization problem solved was

$$
\min \Delta b=\sum_{t=1}^{50} \sum_{i=1}^{5}\left\|b_{t, i}(\mathbf{y})-\hat{b}_{t, i}(\mathbf{y})\right\|_{2}
$$

where

$$
\hat{b}_{t, i}(\mathbf{y})=\mathbf{W}^{H} S_{t}(\mathbf{k}) \mathbf{W} \mathbf{C}_{i} m_{t}\left(\hat{\mathbf{m}}_{0}, \hat{\mathbf{m}}_{c}, \hat{\mathbf{e}}\right)
$$

is a simple extension of Eq12. This arregment is convenient for $2 \mathrm{D}$ sequences where some obels move in adirection normal to the slice.

In the same way as in [12, the displacement of each obel was fitted using periodic quadratic B-Splines with three control points for every spatial direction. Then 

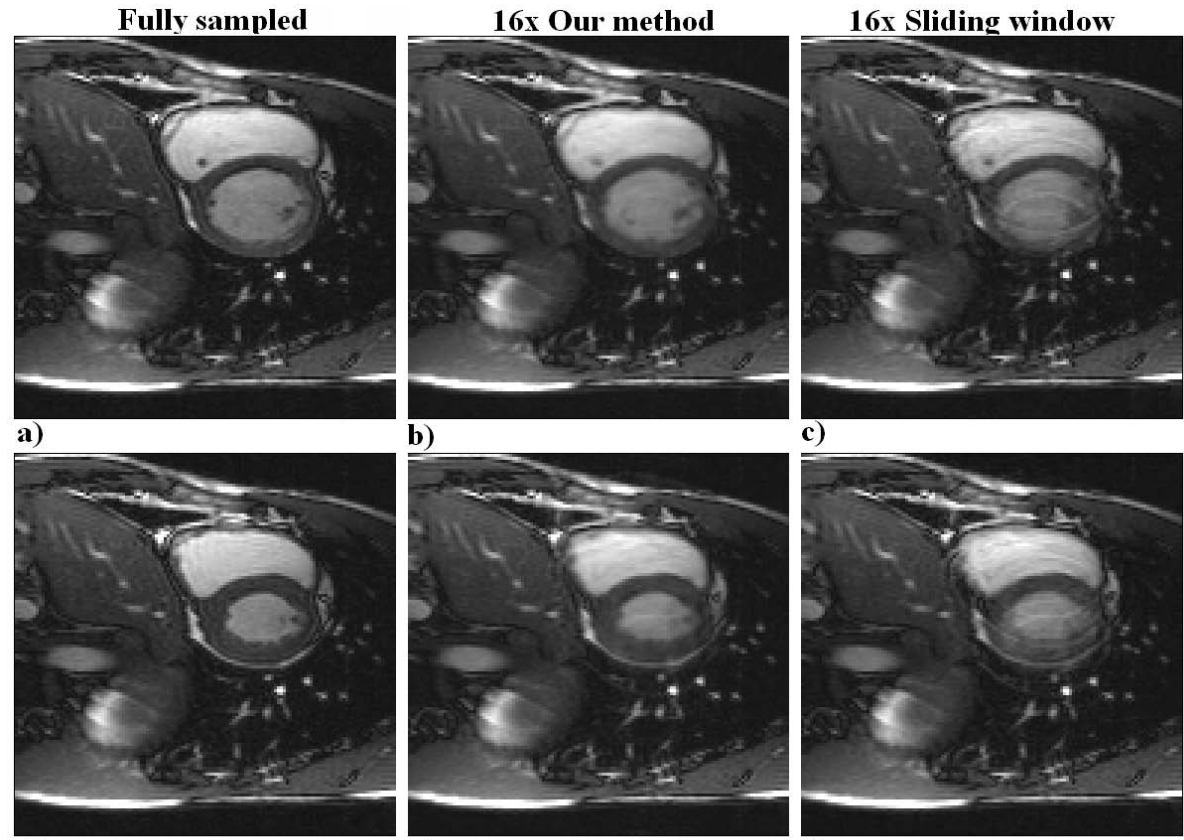

c)

d)

e)

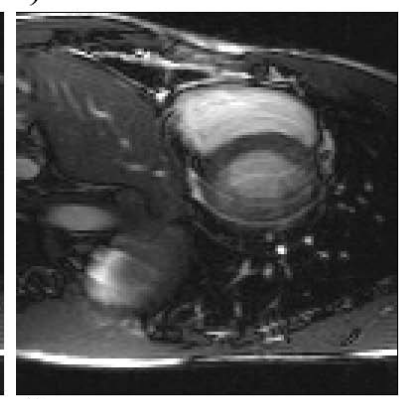

f)

Fig. 4. Reconstructed images from undersampled data using motion estimation. 2D short-axis cardiac sequence with a post-acquisition undersampling factor of 16 . Frames 4 and 27 of a 50-frame sequence are shown. a) Fully sampled frame 4. b) Reconstructed image frame 4 with our method from a 5-coil acquisition. c) Reconstructed image frame 4 with sliding window from a 5-coil acquisition. d) Fully sampled frame 27. e) Reconstructed image frame 27 with our method from a 5-coil acquisition. f) Reconstructed image frame 27 with sliding window from a 5 -coil acquisition.

$$
\mathbf{u}\left(\mathbf{e}_{i}\right)=\sum_{n=0}^{N_{e} / N_{d}-1} \mathbf{e}_{i}^{n} \mathbf{A}^{n}
$$

where $\mathbf{u}\left(\mathbf{e}_{i}\right)$ is the displacement of the obel $i, \mathbf{e}_{i}^{n}$ is the weight applied to the $n^{\text {th }}$ B-Spline base $\mathbf{A}^{n}$ and $N_{e} / N_{d}$ is the number of parameters needed considering one obel per pixel.

The minimization in Eq13 was computed with MATLAB (R2007a, The MathWorks, Natick) routines. The inner loop was solved efficiently using a conjugategradient algorithm (Least Square QR factorization, LSQR). The outer loop was solved employing a trust region method and an aproximation of the analytic gradient of the objetive function. This optimization represents an expensive computational load due to the big amount of unknowns and the non-linear nature of the problem. The reconstruction process took around 6 hours on a regular PC for a image of $128 \times 128$ pixels and 50 time frames. 


\subsection{Results}

The reconstruction results for an undersampling factor of 8 are shown in Fig.2 for two cardiac phases. We have included the fully sampled images (Fig.2a, d), the reconstructed images using our method with multiple-coil acquisition (Fig.2b, e) and the reconstructed images using our method applied to single-coil acquisition (Fig.2c, f). Both reconstructions are in good agreement with the fully sampled image, with root mean square (RMS) errors of $2.65 \%$ and $2.45 \%$ for single and muliple coil, respectively. A zooming in on the differences between the fully sampled and the reconstructed images for a particular frame is shown in Fig.3. The difference images show that the main errors are due to small displacement of the edges, reaching a better estimation using the multiple coil approach.

The reconstruction results for an undersampling factor of 16 are shown in Figs.4 and 5. We have included in Fig. 4 two selected fully sampled time frames (Fig.4a, d), the corresponding reconstructions using our method applied to multiple-coil acquisition (Fig.4b, e) and the corresponding reconstructions using SW (Fig.4c, f). The image sequence reached with the proposed method has an RMS error of $1.72 \%$ compared to the fully sampled image, while the one reconstructed with SW has an RMS error of $2.03 \%$. These results show that most of the aliasing was eliminated and only a slight spatial and temporal blurring remains, which is dependent on the quality of the reference frames. The temporal blurring is more evident in Fig.5 which shows the evolution over time of a line

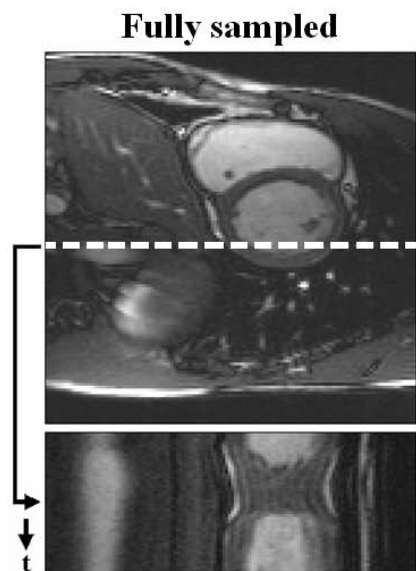

a) 16x Our method

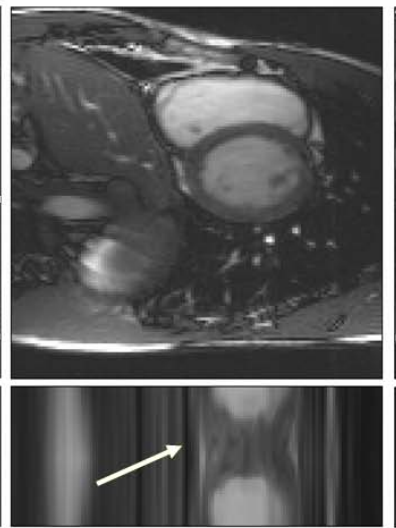

b)

\section{6x Sliding window}

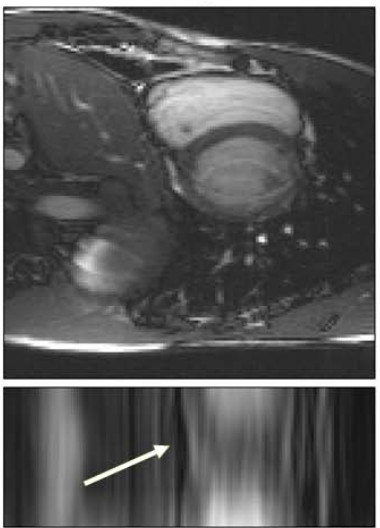

c)

Fig. 5. Time evolution. 2D short-axis cardiac sequence with a post-acquisition undersampling factor of 16 . The images show the temporal evolution of a line passing through the left ventricle. a) Temporal evolution for the fully sampled image. b) Temporal evolution for the reconstructed image using our method from a 5-coil acquisition. c) Evolution temporal for the reconstructed image using sliding window from a 5-coil acquisition. 
passing through the left ventricle. Again, we have included the temporal evolution for the fully sampled sequence and the reconstructions using our method and SW.

\section{Summary and Conclusions}

An application of motion estimation to reconstruct undersampled dynamic MRI was presented. This is an extension of the method based on modeling the motion of objects elements (obels) to parallel imaging using multiple coils. Further undersampling factor and/or improved reconstruction accuracy is possible through the proposed method. It was demonstrated using $2 \mathrm{D}$ cardiac images acquired with 5 coils and undersampling factors of 8 and 16 (i.e. acquisitions are 8 and 16 times faster, respectively). For an undersampling factor of 8 images reconstructed with the proposed method display better quality than those obtained using only one coil with the same undersampling. An undersampling factor of 16 is quite feasible using multiple coils. This level of undersampling is not possible using one coil because of constraints in the amount of data. The proposed method does not requiere the motion to be confined to a portion of the field of view or to a portion of the temporal frequency. Moreover, an approximation of the vector field of motion is obtained as an additional result.

\section{References}

1. Abd-Elmoniem, K.Z., Osman, N.F., Prince, J.L., Stuber, M.: Three-dimensional magnetic resonance myocardial motion tracking from a single image plane. Magn. Reson. Med. 58, 92-102 (2007)

2. Sakuma, H.: Magnetic resonance imaging for ischemic heart disease. J. Magn. Reson. Imaging. 26, 3-13 (2007)

3. Raman, V.K., Lederman, R.J.: Interventional cardiovascular magnetic resonance imaging. Trends Cardiovasc. Med. 17, 196-202 (2007)

4. Gupta, V., Khandelwal, N., Mathuria, S., Singh, P., Pathak, A., Suri, S.: Dynamic magnetic resonance imaging evaluation of craniovertebral junction abnormalities. J. Comput. Assist. Tomogr. 31, 354-359 (2007)

5. Xiang, Q.S., Henkelman, R.M.: k-space description for MR imaging of dynamic objects. Magn. Reson. Med. 29, 422-428 (1993)

6. Jones, R.A., Haraldseth, O., Muller, T.B., Rinck, P.A., Oksendal, A.N.: k-space substitution: a novel dynamic imaging technique. Magn. Reson. Med. 29, 830-834 (1993)

7. van Vaals, J.J., Brummer, M.E., Dixon, W.T., Tuithof, H.H., Engels, H., Nelson, R.C., Gerety, B.M., Chezmar, J.L., den Boer, J.A.: Keyhole method for accelerating imaging of contrast agent uptake. J. Magn. Reson. Imaging 3, 671-675 (1993)

8. Hu, X., Parrish, T.: Reduction of field of view for dynamic imaging. Magn. Reson. Med. 31, 691-694 (1994)

9. Madore, B., Glover, G.H., Pelc, N.J.: Unaliasing by Fourier-encoding the overlaps using the temporal dimension (UNFOLD), applied to cardiac imaging and fMRI. Magn. Reson. Med. 42, 813-828 (1999) 
10. Tsao, J., Boesinger, P., Pruessmann, K.P.: k-t BLAST and k-t SENSE: dynamic MRI with high frame rate exploiting spatiotemporal correlations. Magn. Reson. Med. 50, 1031-1042 (2003)

11. Irarrazaval, P., Bourbetakh, R., Razavi, R., Hill, D.: Dynamic three-dimensional undersampled data reconstruction employing temporal registration. Magn. Reson. Med. 54, 1207-1215 (2005)

12. Prieto, C., Batchelor, P., Hill, D., Hajnal, J., Guarini, M., Irarrazaval, P.: Reconstruction of undersampled dynamic images by modeling the motion of objects elements. Magn. Reson. Med. 57, 939-949 (2007)

13. Pruessmann, K.P., Werger, M., Scheidegger, M.B., Boesinger, P.: SENSE: Sensitivity encoding for fast MRI. Magn. Reson. Med. 42, 952-962 (1999)

14. Sodickson, D.K., Mannin, W.J.: Simultaneous acquisition of spatial harmonics (SMASH): ultra-fast imaging with radiofrequency coil arrays. Magn. Reson. Med. 38, 591-603 (1997)

15. Batchelor, P.G., Atkinson, D., Irarrazaval, P., Hill, D.L.G., Hajnal, J., Larkman, D.: Matrix description of genearal motion correction applied to multishot images. Magn. Reson. Med. 54, 1273-1280 (2005)

16. d'Arcy, J.A., Collins, D.J., Rowland, I.J., Padhani, A.R., Leach, M.O.: Applications of sliding window reconstruction with Cartesin sampling for dynamic contrast enhancement MRI. NMR. BioMed. 15, 174-183 (2002) 